\title{
Sleep disorders in multiple sclerosis: present-day knowledge and future perspectives
}

Keywords: multiple sclerosis, sleep, fatigue, mood, demyelination, psychiatric manifestations

\section{Introduction}

Multiple sclerosis (MS) is a chronic inflammatory and neurodegenerative disease of the central nervous system characterized by demyelination and axonal loss. It usually occurs in individuals aged between 20 and 40 years and constitutes the first cause of nontraumatic disability in young adults. Through the disease course, patients may suffer from a plethora of symptoms including sensory, motor, cerebellar complaints, but also fatigue, cognitive deficits, psychiatric manifestations and sleep disturbances. Fatigue could be a very debilitating and difficult-to-treat symptom with a prevalence reaching up to $90 \% .^{1,2}$ Psychiatric comorbidities, namely anxiety and depression, might occur in up to $95 \%$ of patients and could even herald MS diagnosis. ${ }^{3}$ Cognitive deficits can affect about $65 \%$ of patients. ${ }^{4,5}$ Importantly, sleep disturbances are commonly reported by MS patients where they could occur approximately four times more than in the general population. ${ }^{1,6}$ Sleep disturbances could be either due to co-morbid sleep disorders or secondary to several factors that frequently happen in MS. To start, regarding comorbid sleep disorders, the occurrence of restless leg syndrome has been found to be 5.4 times greater in MS patients compared to the general public and seems to arise from hypothalamic and cervical spine lesions. ${ }^{1}$ Sleep apnea is another condition that may be related to brainstem lesions or medication-induced hypotonia of pharyngeal muscles. ${ }^{1}$ Rapid eye movement behavior disorders could also arise in MS patients and seem to be linked to dorsal pontine lesions. ${ }^{1}$ Other encountered sleep syndromes include periodic limb movements and narcolepsy-cataplexy. ${ }^{1}$ Furthermore, the restorative sleep capacity may be altered in this population as a consequence to functional and pathological changes of the disease itself, such changes would lead to excessive daytime sleepiness. ${ }^{1}$ It is worth noting that, in a recent functional MRI study, patients with disturbed sleep pattern exhibited decreased functional connectivity between the thalamus and some frontoparietal areas compared to those with normal sleep pattern. ${ }^{7}$ Besides sleep disorders, several factors frequently observed in MS may precipitate this problem. These mainly include bladder dysfunction, with nocturia being the major contributor to middle and terminal insomnia; ${ }^{1}$ and lower limb dysesthesias, which result from MS-related pathological changes in central sensory pathways and can affect up to $86 \%$ of patients. ${ }^{1}$ It is also of great importance to consider the interaction between sleep and each of fatigue and mood. ${ }^{8}$ Particularly, MS patients with poor sleep quality were found to have higher fatigue and depression scores. ${ }^{9}$ Moreover, compared to patients without anxiety, those with anxiety were about two times more prone to experience sleep troubles. ${ }^{6}$ In addition to these comorbidities, and despite the little available evidence, some disease modifying treatments (i.e. interferon-beta) and symptomatic drugs may affect sleep quality. Conversely, other treatments, such as natalizumab, may ameliorate sleep. ${ }^{10}$ The paucity of studies with regards to this topic warrants further research. In conclusion, the available data hint toward a relatively high prevalence of sleep disturbances in MS
Volume I Issue I - 2017

\author{
Samar S Ayache, ,2, 3 Moussa A Chalah ${ }^{1,2}$ \\ 'Excitabilité Nerveuse et Thérapeutique, Université Paris-Est- \\ Créteil, France \\ ${ }^{2}$ Service de Physiologie-Explorations Fonctionnelles, Hôpital \\ Henri Mondor, France \\ ${ }^{3}$ Lebanese American University Medical Center, Rizk hospital \\ (LAUMC-RH), Lebanon
}

Correspondence: Samar Ayache, Service de PhysiologieExplorations Fonctionnelles, Hôpital Henri Mondor, Assistance Publique-Hôpitaux de Paris, 940 I0, Créteil, France. Email samarayache@gmail.com

Received: July 30, 2017 | Published: July 31, 2017

patients. Admitting the drastic impact of sleep disturbance on physical and mental well-being, ${ }^{6}$ a systematic screening for sleep disorders in daily clinical practice should be implemented by adapting short quantitative assessment tools such as Epworth Sleepiness Scale. ${ }^{1}$ This would allow a proper referral of concerned patients to sleep specialists for further investigations (e.g. polysomnography). Considering the frequent occurrence of comorbidities and their relationship with sleep quality, it would be of value to evaluate and treat fatigue (e.g. using the Modified Fatigue Impact Scale), mood disorders (e.g. by means of Hospital Anxiety and Depression Scale) and neuropathic pain (e.g. via the Neuropathic Pain Inventory), which may aggravate patients' sleep problems. ${ }^{1}$ Finally, sleep disorders merit to be addressed in future works for a better understanding of these pathologies and their relationships with MS comorbidities, in order to optimize therapeutic strategies and improve patients' quality of life.

\section{Declaration of interest}

This work did not receive any specific grant. MAC declares no conflict of interest. SSA declares having received travel grants or compensation from Genzyme, Biogen, Novartis and Roche.

\section{Acknowledgements}

None.

\section{Conflict of interest}

The author declares no conflict of interest.

\section{References}

1. Ayache SS, Chalah MA. Fatigue in multiple sclerosis-insights into evaluation and management. Neurophysiol Clin. 2017;47(2):139-171.

2. Chalah MA, Riachi N, Ahdab R, et al. Fatigue in multiple sclerosis: neural correlates and the role of non-invasive brain stimulation. Front Cell Neurosci. 2015;9:460.

3. Chalah MA, Ayache SS. Psychiatric presentation in multiple sclerosis: could it be the tip of the iceberg? Rev Bras Psiquiatr. 2017. S1516 p. 
4. Ayache SS, Palm U, Chalah MA, et al. Orienting network dysfunction in progressive multiple sclerosis. J Neurol Sci. 2015;351(1-2):206-207.

5. Chalah MA, Ayache SS. Deficits in social cognition: an unveiled signature of multiple sclerosis. J Int Neuropsychol Soc. 2017;23(3):266-286.

6. Garland SN, Scurrey SRM, Ploughman M. Health, Lifestyle and aging with ms canadian consortium. Factors associated with poor sleep in older adults with multiple sclerosis. Int J Behav Med. 2017.

7. Van Geest Q, Westerik B, van der Werf YD, et al. The role of sleep on cognition and functional connectivity in patients with multiple sclerosis. $J$ Neurol. 2017;264(1):72-80.
8. Veauthier C, Gaede G, Radbruch H, et al. Poor sleep in multiple sclerosis correlates with beck depression inventory values, but not with polysomnographic data. Sleep Disord. 2016;2016:8378423.

9. Nociti V, Losavio FA, Gnoni V, et al. Sleep and fatigue in multiple sclerosis: A questionnaire-based, cross-sectional, cohort study. J Neurol Sci. 2017;372:387-392.

10. Lanza G, Ferri R, Bella R, et al. The impact of drugs for multiple sclerosis on sleep. Mult Scler. 2017;23(1):5-13. 